Document downloaded from:

http://hdl.handle.net/10251/104246

This paper must be cited as:

Del Canto, I.; Lopez-Grueso, R.; Gambini, J.; Monleón, D.; Borrás, C.; Viña, J.; Moratal, D. (2015). PETra: Software Tool for a Semiautomatic Positron Emission Tomography Image Analysis and its Application to the Study of Brain Glucose Consumption in Rats. IEEE Latin America Transactions. 13(3):876-884. doi:10.1109/TLA.2015.7069118

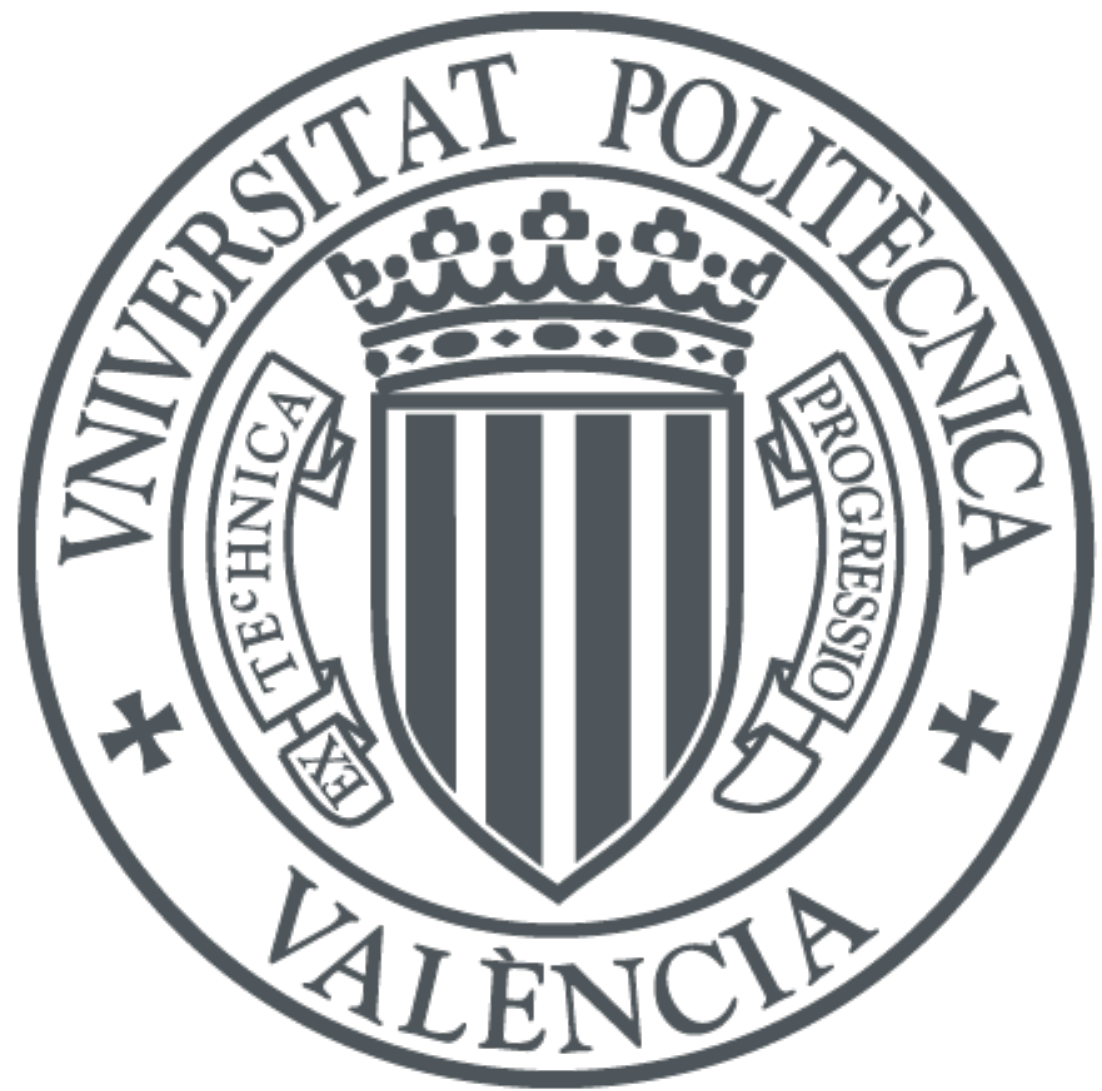

The final publication is available at

https://doi.org/10.1109/TLA.2015.7069118

Copyright Institute of Electrical and Electronics Engineers

Additional Information 


\title{
PETra: Software Tool for a Semiautomatic Positron Emission Tomography Image Analysis and its Application to the Study of Brain Glucose Consumption in Rats
}

\author{
I. Del Canto, R. L. Grueso, J. Gambini, D. Monleón, C. Borrás, J. Viña and D. Moratal, Senior \\ Member, IEEE
}

\begin{abstract}
This work presents a Positron Emission Tomography (PET) image analysis tool and its application to the study of rat brain glucose consumption (PETra comes from PET+rat). The described methodology has four steps: a preprocessing of PET images, a coregistration of these images with an atlas, a semiautomatic segmentation of the regions of interest in the rat brain and a $3 \mathrm{D}$ reconstruction of these regions to obtain the volumes of interest. Brain glucose uptake was quantified as Standardized Uptake Value (SUV). This tool was applied to nine Wistar rats, young (4-7 months) and old (22-24 months) groups, to study the effect of aging on brain glucose consumption and the difference between sexes. Results showed a lower glucose uptake in old rats than in young rats, regardless gender; while young female rats showed higher glucose consumption than young male rats, whereas these differences disappeared with aging. The developed tool allows the quantification of glucose in rat brain. Results show the accuracy of the tool to define ranges of variation in a population of young and old rats, showing a decrease in glucose consumption in aging.
\end{abstract}

Keywords - coregistration, segmentation, brain Standardized Uptake Value, Positron Emission Tomography.

\section{INTRODUCCIÓN}

$\mathrm{L}$ A TOMOGRAFÍA por emisión de positrones (PET)

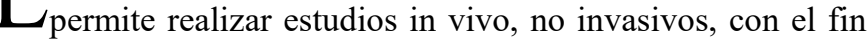
de medir la actividad metabólica de los diferentes tejidos en humanos o en animales, especialmente del sistema nervioso central.

De hecho, por ser la glucosa uno de los principales sustratos energéticos del organismo y más concretamente del cerebro, el radiotrazador más empleado en tomografía por emisión de positrones (PET) para los estudios que conciernen al cerebro es la 2-[18F]-fluoro-2-desoxi-D-glucosa $\left({ }^{18} \mathrm{~F}-\mathrm{FDG}\right)$.

I. Del Canto, Fundación de Investigación del Hospital Clínico Universitario de Valencia, INCLIVA, Valencia, España, dmoratal@eln.upv.es

R. L. Grueso, Fundación de Investigación del Hospital Clínico Universitario de Valencia, INCLIVA, Valencia, España, Raul.lopez@uv.es

J. Gambini, Fundación de Investigación del Hospital Clínico Universitario de Valencia, INCLIVA, Valencia, España, Juan.Gambini@uv.es

D. Monleón, Fundación de Investigación del Hospital Clínico Universitario de Valencia, INCLIVA, Valencia, España, daniel.monleon@uv.es

C. Borrás, Fundación de Investigación del Hospital Clínico Universitario de Valencia, INCLIVA, Valencia, España, Consuelo.Borras@uv.es

J. Viña, Fundación de Investigación del Hospital Clínico Universitario de Valencia, INCLIVA, Valencia, España, Jose.Vina@uv.es

D. Moratal, Centro de Biomateriales e Ingeniería Tisular de la Universitat Politècnica de València, Valencia, España, dmoratal@eln.upv.es
Las imágenes obtenidas a partir de este radiotrazador permiten la localización y la cuantificación de la captación de glucosa [1] por las diferentes áreas cerebrales, ofreciendo un arma de capital importancia a la investigación biomédica y al diagnóstico médico, ya que muestra qué áreas del cerebro tienen un metabolismo glucídico incrementado o reducido.

La distribución cerebral de ${ }^{18} \mathrm{~F}-\mathrm{FDG}$ y las variaciones en la intensidad de captación de la misma han sido objeto de numerosas investigaciones. Estudios previos realizados con ${ }^{18}$ F-FDG-PET en cerebro abarcan varias áreas incluyendo enfermedades neurodegenerativas, neurooncología y envejecimiento. En estudios relacionados con la demencia tipo Alzhéimer, se ha detectado hipometabolismo de FDG en diferentes áreas de la corteza cerebral, en un estadio muy precoz de la enfermedad [2], así como reducciones del metabolismo cerebral de glucosa en individuos con historial materno de dicha enfermedad [3]. Por el contrario, en estudios con enfermos de Parkinson, se ha hallado un incremento del metabolismo de la glucosa, también en estadios tempranos [4]. En neurooncología, se ha utilizado la imagen ${ }^{18}$ F-FDG-PET para discriminar tumores de alto grado y bajo grado de malignidad, establecer factores pronósticos, seleccionar el lugar óptimo para la obtención de una biopsia y establecer el diagnóstico diferencial entre recurrencia y radionecrosis en tumores ya tratados [5]. En el envejecimiento cerebral, se ha observado una disminución en el metabolismo cerebral de glucosa con el envejecimiento [6], siendo varios los estudios que han detectado este menor consumo de glucosa en algunas regiones cerebrales concretas como el cingulado anterior [7] o la región hipocampal [8]. Con el fin de analizar las variaciones mencionadas, se han realizado estudios funcionales in vivo con animales de laboratorio [9], [10].

La captación cerebral de ${ }^{18} \mathrm{~F}-\mathrm{FDG}$ puede ser evaluada de manera no invasiva a través del Standardized Uptake Value (SUV), índice semicuantitativo que relaciona la concentración del radiofármaco en un órgano o lesión analizada mediante una región de interés (ROI) con la actividad inyectada y el peso corporal del sujeto, método que goza de valor adicional a la interpretación cualitativa visual de la captación del radiofármaco [11].

La técnica PET ha evolucionado y se ha perfeccionado, siendo ampliamente utilizada en la investigación biomédica, concretamente en el ámbito de la neurología [12]. Sin 
embargo, es cierto que tanto la reconstrucción de las imágenes PET [13] como la metodología utilizada para el análisis y cuantificación de las mismas [14] todavía plantean dudas.

Las imágenes moleculares suelen tener menor resolución que las imágenes anatómicas correspondientes $\mathrm{y}$, adicionalmente, no siempre es posible obtener dicha imagen anatómica por lo que en ocasiones es necesario recurrir al análisis de la imagen molecular en si misma, sin una referencia anatómica precisa. Además, la cuantificación de la señal de las regiones de interés correspondientes, seleccionadas de modo manual, está sujeta a importantes variaciones subjetivas. Por último, el acceso limitado a las imágenes anatómicas hace que en numerosas ocasiones la comparación de resultados entre individuos resulte extremadamente compleja.

En este trabajo se presenta una herramienta-software para el análisis semiautomático de las imágenes moleculares que permite la detección y cuantificación de regiones de interés en el cerebro de rata, coregistrando estas imágenes con un atlas anatómico, con el fin de estudiar el consumo de glucosa en el envejecimiento, comparando la captación cerebral de ${ }^{18} \mathrm{~F}-\mathrm{FDG}$ en ratas jóvenes y viejas. La automatización de esta cuantificación permitirá anular la variabilidad interobservador, evitando el error perceptual del investigador.

\section{MATERIAL Y MÉTODOS}

\section{A. Grupo de estudio}

Se llevó a cabo un estudio experimental en el que se incluyeron 9 ratas Wistar: 5 ratas hembra (3 jóvenes y 2 viejas) y 4 ratas macho ( 2 jóvenes y 2 viejas). Las edades de los animales estaban comprendidas entre 4 y 7 meses para el grupo de ratas jóvenes y entre 22 y 24 meses para el grupo de ratas viejas. El estudio se realizó de acuerdo con lo establecido en el Real Decreto 1201/2005, del 10 de octubre, sobre protección de los animales utilizados para experimentación y otros fines científicos (BOE, 21 de octubre de 2005) [15].

\section{B. Preparación experimental y adquisición de las imágenes}

Los estudios PET de cuerpo entero con ${ }^{18} \mathrm{~F}-\mathrm{FDG}$ se llevaron a cabo con un equipo PET (Albira ONCOVISION, GEM-Imaging, Valencia, España). Se mantuvo a los animales entre 8 y $14 \mathrm{~h}$ en ayuno antes de la anestesia con isoflurano $(1,5-2 \%$ en $100 \%$ de oxígeno, IsoFLo; Abbott Laboratories) y de la inyección intravenosa de una dosis de entre 30 y $40 \mathrm{MBq}$ (entre 0,811 y $1,081 \mathrm{mCi})$ de ${ }^{18} \mathrm{~F}-\mathrm{FDG}$ [16], sintetizado como se describe en [17]. Tras completar un periodo de distribución del radiotrazador de 40 minutos, el escáner inicia la adquisición. El equipo PET utilizado consiste en un tomógrafo con detectores continuos de cristal centelleador LYSO acoplados a tubos fotomultiplicadores sensibles a la posición (PS-PMT). La configuración es octogonal y existe detección de profundidad de interacción (DOI) medida a partir de la distribución del haz de fotones ópticos detectados en el PS-PMT [18]. La resolución espacial es de 1,6 mm, y la sensibilidad absoluta de $0,7 \%$.

Las imágenes de emisión fueron adquiridas mediante 10 secuencias de 2 minutos cada una ( 2 minutos por posición de camilla). Posteriormente, se generaron las imágenes PET usando un algoritmo de reconstrucción tridimensional con corrección por decaimiento (puesto que la ${ }^{18} \mathrm{~F}$-FDG tiene una vida media de 109,8 minutos). A partir de cada una de estas imágenes tridimensionales, se extrajeron 72 cortes axiales, sagitales y coronales. Se obtuvieron por tanto series de 72 imágenes PET en formato DICOM de 16 bits con una resolución de $72 \times 72$ píxeles, correspondientes a cortes axiales. Se analizaron un total de nueve secuencias de 72 imágenes PET (imagen DICOM) de las nueve ratas del estudio. En la Fig. 1 (a,b,c), se representan 3 imágenes PET de cortes transversales del cerebro de una rata Wistar hembra joven.

\section{Atlas estandarizado}

Con el fin de ubicar de forma precisa la anatomía de las imágenes PET, se recurrió a un atlas estandarizado de cerebro de rata [22]. El atlas de cerebro consiste en una serie de mapas que representan una estructura bidimensional de una sección cerebral en cualquiera de las tres orientaciones anatómicas (axial, sagital y coronal). Con la ayuda de este atlas, y tras corregistrarlo correctamente con la imagen de PET cerebral de la rata, se localizaron e identificaron las regiones de interés (ROIs) y los contornos del cerebro. Por lo tanto, el atlas permitió localizar una ROI en las imágenes de PET de distintos cerebros, $\mathrm{y}$ de este modo la comparación entre valores obtenidos de su cuantificación. En la Fig. 1 (d,e,f), se representan 3 imágenes de atlas de cortes transversales de cerebro de rata.

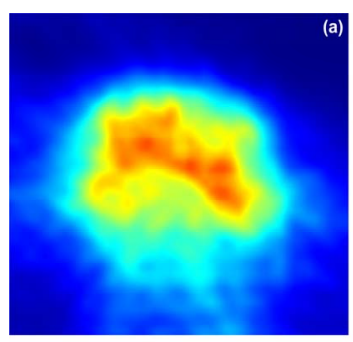

(d)
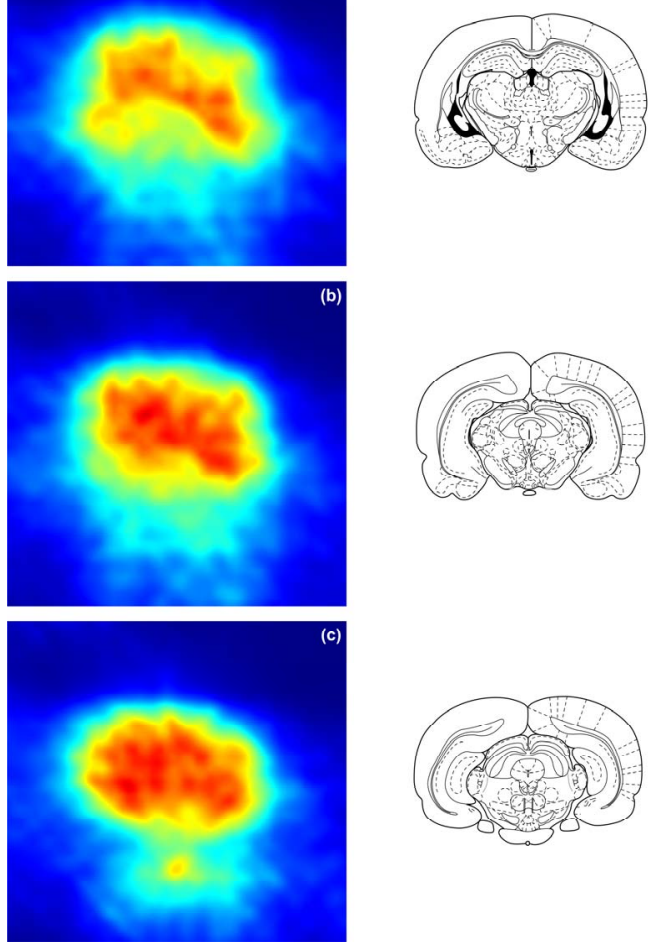

(†)
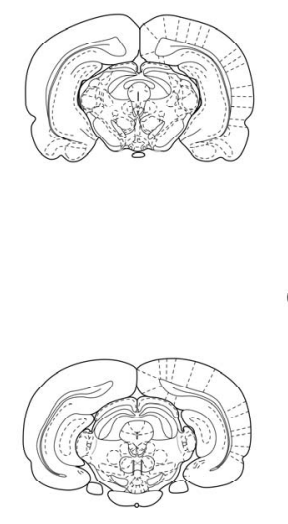

Figura 1. $(\mathrm{a}, \mathrm{b}, \mathrm{c})$ Imágenes PET de 3 cortes transversales sucesivos del cerebro de una rata Wistar hembra joven y $(\mathrm{d}, \mathrm{e}, \mathrm{f})$ sus imágenes de atlas correspondientes. 


\section{Procesado de imagen}

Todas las imágenes en formato DICOM provenientes del equipo PET se convirtieron a escala de gris y se redimensionaron a imágenes de mayor tamaño $(740 \times 740$ píxeles) para facilitar la delimitación de las regiones de interés. Las imágenes de atlas en formato digital de [19] se invirtieron para superponerlas a las imágenes de PET y poder visualizar los contornos del atlas y de la región de interés con facilidad. En la Fig. 2 (a y b) se muestra el resultado de la aplicación de dichas transformaciones. Estas imágenes fueron el punto de partida de cada uno de los procesos posteriores.
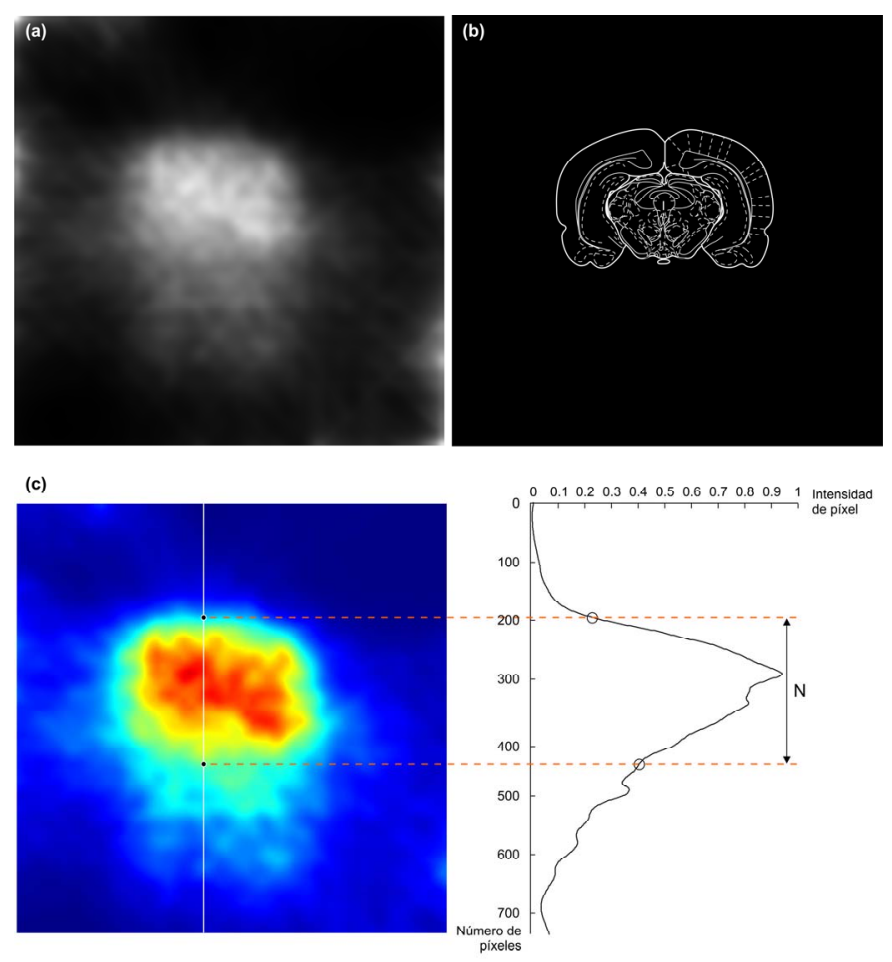

Figura 2. Procesado previo de las imágenes (a) Imagen DICOM redimensionada a $740 \times 740$ píxeles y normalizada. (b) Imagen binarizada del atlas (umbral $=0,9$ ) e invertida. (c) Perfil de contraste (a lo largo de la línea blanca vertical) y selección de los puntos que determinan el tamaño del cerebro para el ajuste del atlas.

\section{E. Corregistro del atlas a las imágenes PET}

\section{Ajuste del atlas al cerebro mediante perfil de contrastes}

El corregistro del atlas con la imagen PET requirió de un ajuste previo de dicho atlas al tamaño del cerebro de la rata, realizado mediante un perfil de contrastes. En una imagen, el perfil es una representación del valor de los distintos píxeles de una imagen a lo largo de una línea que la atraviesa. En las imágenes DICOM, definimos los límites del cerebro en sentido vertical sirviéndonos del perfil de contrastes $\mathrm{y}$, a partir de éstos, se obtuvo el tamaño (vertical) del cerebro $(N)$ para, posteriormente, ajustar el tamaño del atlas al mismo de forma automática (Fig. 2c). Las dimensiones del atlas ajustado, $p$ (vertical) y $q$ (horizontal), se calculan a partir de las dimensiones del atlas inicial, $r$ (vertical) y $s$ (horizontal):

$$
\begin{aligned}
& p=N \\
& q=s \cdot N / r
\end{aligned}
$$

\section{Superposición atlas - imagen PET}

El corregistro se llevó a cabo mediante una superposición de las dos imágenes. Además, se requirió cierta transparencia entre atlas e imagen PET que consistía en 3 pasos: 1) representar la imagen original (imagen PET), 2) superponer una imagen de color sólido (blanco) sobre la imagen original, 3) utilizar los píxeles de otro conjunto de datos llamado mapa de influencia (imagen atlas) para variar la transparencia de la imagen de color sólido píxel a píxel (Fig. 3).
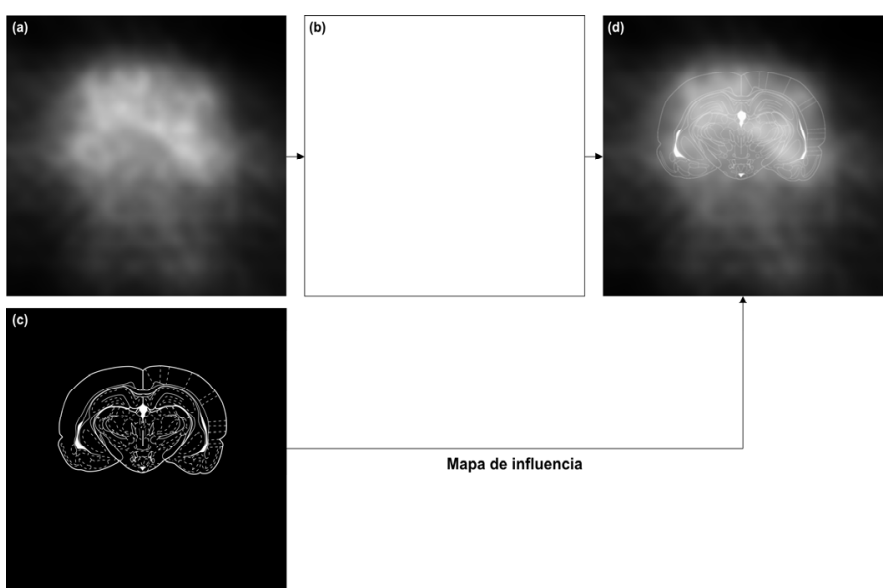

Figura 3. Superposición de la imagen PET a su atlas correspondiente mediante la técnica de transparencia. La superposición se realiza en 3 etapas: 1) representar la imagen PET (a); 2) superponer la imagen de color sólido blanco (b) sobre la imagen PET; 3) utilizar los píxeles del atlas (c) como mapa de influencia para controlar la transparencia de cada píxel de la imagen blanca, y obtener la superposición (d).

\section{Transformaciones afines}

El corregistro se basó en diversas transformaciones afines (escalado, traslación y rotación) para localizar y desplazar el atlas sobre la imagen PET para identificar las ROIs.

\section{a) Escalado}

El escalado aplicado al atlas fue uniforme, es decir, un único factor para ambas dimensiones, y se utilizó el método de interpolación cúbica.

$$
I_{E S C}=I_{O R I G} \cdot E(\lambda) \quad E(\lambda)=\left(\begin{array}{ccc}
\lambda & 0 & 0 \\
0 & \lambda & 0 \\
0 & 0 & 1
\end{array}\right)
$$

donde $I_{E S C}$ es la imagen escalada, $I_{O R I G}$ es la imagen origen y $\lambda$ es el factor de escala.

\section{b) Traslación}

La traslación se realizó mediante técnicas de enventanado (Fig. 4): partiendo de una matriz de ceros del tamaño de la imagen adquirida $(m \times n)$, se desplaza en su interior el atlas previamente ajustado $(p \times q)$ en función de los desplazamientos horizontales $(x)$ y verticales $(y)$, así como de 
la ordenada del punto inicial del cerebro (ay), según (4):

$$
I\left(a y+y: a y+p-1+y, \frac{n}{2}-\frac{q}{2}+x: \frac{n}{2}+\frac{q}{2}-1+x\right)=A
$$

donde $I$ es la matriz de ceros $(m \times n)$ y $A$ es la matriz correspondiente al atlas ajustado $(p \times q)$.

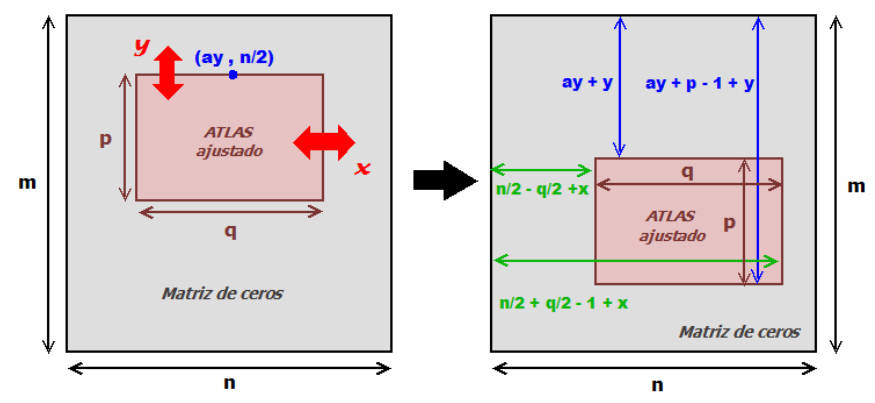

Figura 4. Traslación del atlas mediante técnicas de enventanado $(x>0$ : hacia la derecha, $x<0$ : hacia la izquierda; $y>0$ : hacia abajo, $y<0$ : hacia arriba).

\section{c) Rotación}

La rotación de una imagen un determinado ángulo tiene la siguiente representación matricial:

$$
I_{R O T}=I_{O R I G} \cdot R(\theta) \quad R(\theta)=\left(\begin{array}{ccc}
\cos \theta & \sin \theta & 0 \\
-\sin \theta & \cos \theta & 0 \\
0 & 0 & 1
\end{array}\right)
$$

donde $I_{R O T}$ es la imagen rotada, $I_{O R I G}$ es la imagen origen y $\theta$ es el ángulo de giro.

No obstante, cuando se trabaja con imágenes, se utiliza la imagen origen para rellenar los píxeles de la imagen rotada, creada previamente sin ningún contenido, aplicándole a esta última la inversa de la transformación descrita en (5), es decir:

$$
\left(\begin{array}{c}
i^{\prime} \\
j^{\prime} \\
1
\end{array}\right)=\left(\begin{array}{ccc}
\cos \theta & -\sin \theta & 0 \\
\sin \theta & \cos \theta & 0 \\
0 & 0 & 1
\end{array}\right)\left(\begin{array}{l}
i \\
j \\
1
\end{array}\right)
$$

donde $\left(i^{\prime}, j^{\prime}\right)$ son las coordenadas de un punto en la imagen origen, y $(i, j)$ son las coordenadas de un punto en la imagen rotada.

De este modo, cuando estemos hallando a qué píxel corresponde el punto $(i, j)$ de la imagen rotada en la imagen origen, la inversa nos dará un punto $\left(i^{\prime}, j^{\prime}\right)$ de la imagen origen, donde $i^{\prime}$ y $j^{\prime}$ no serán necesariamente enteros. Esto nos obliga a tener que realizar una interpolación sobre los valores de entrada para generar los valores de salida. $\mathrm{La}$ interpolación fue bicúbica, a partir del promedio de 16 píxeles adyacentes. Por último, a los puntos de la imagen rotada que no corresponden a ningún punto de la imagen origen, se les asignó el valor mínimo de la imagen, es decir, el 0 o color negro.

\section{F. Segmentación de ROIs}

El proceso de segmentación permite automatizar la delimitación de las distintas regiones de interés del atlas, evitando la tediosa tarea de su selección manual en cada una de las imágenes, definiendo estas áreas con tan sólo seleccionar un punto en el centro de la ROI del atlas. Este punto inicial marcado con el ratón sirvió de semilla para la estimación de un contorno que toma como entrada un modelo de contorno activo, o snakes [20], y que segmenta, tras un determinado número de iteraciones, la región de interés seleccionada, de forma automática.

\section{Estimación de un contorno inicial}

La estimación de un contorno inicial, primera etapa del algoritmo, se basó en la aplicación de un umbral de binarización de valor próximo a 0,9 sobre la imagen de atlas seleccionada y la posterior inversión de la imagen binarizada. A continuación, se obtuvo el contorno inicial a partir de la información proporcionada por el usuario al seleccionar un punto en el interior de la región a segmentar: el algoritmo detectó cuatro puntos correspondientes a los desplazamientos según $x,-x, y$ y $-y$ desde el punto inicial, imponiendo como criterio de parada la detección de un punto blanco, es decir, de un contorno. El resultado final fue la curva que serviría como contorno inicial del modelo deformable o snake (Fig. 5b).

\section{Ajuste final}

El ajuste final del contorno se realizó mediante la técnica de contornos activos (o snakes) del flujo del vector gradiente o gradient vector flow (GVF) [21]. El snake es una curva elástica, que puede deformarse debido a las fuerzas externas que la atraen hacia las características más destacadas de la imagen (bordes) y a las fuerzas internas que intentan preservar la suavidad en la forma de la curva. En estos modelos, se busca obtener una curva que minimice la función de energía:

$$
E_{\text {snake }}(V)=\sum_{i=1}^{n} E_{\text {int }}\left(v_{i}\right)+\kappa \cdot E_{\text {ext }}\left(v_{i}, I\right)
$$

donde $V=\left[v_{1}, \ldots, v_{n}\right]$ define los puntos de la curva, $v_{i}=\left(x_{i}, y_{i}\right)$, $x$ e $y$ son las coordenadas de la curva, $\kappa$ es un factor de ponderación, $E_{\text {snake }}$ es la energía total asociada al snake, $E_{\text {int }}$ es la energía asociada a la curva en sí misma y $E_{e x t}$ es la energía asociada a las características de la imagen $I$.

La energía interna se determina a partir de las características de la curva (o contorno), de ahí la necesidad de haber estimado un contorno inicial previamente que pueda deformarse buscando minimizar la función de energía. El algoritmo de deformación del snake utiliza un número dado de parámetros que determina cómo se comportará internamente la curva, es decir, su elasticidad (alfa, $\alpha$ ), rigidez (beta, $\beta$ ), viscosidad (gamma, $\gamma$ ) y peso de la fuerza de presión (presión, $\kappa)$, sin considerar de momento las características de la imagen.

Si $V$ representa la curva deformable, la energía interna se puede reescribir como: 


$$
E_{\text {srake }}(V)=\sum_{i=1}^{n} \alpha \cdot\left|V^{\prime}\left(v_{i}\right)\right|^{2}+\beta \cdot\left|V^{\prime \prime}\left(v_{i}\right)\right|^{2}
$$

donde $V^{\prime}\left(v_{i}\right)$ y $V^{\prime \prime}\left(v_{i}\right)$ representan la primera y segunda derivadas, respectivamente, y $\alpha$ (elasticidad) y $\beta$ (rigidez) son los pesos correspondientes.

El parámetro de viscosidad se utiliza en la implementación iterativa para controlar la rapidez con la que la curva puede deformarse entre iteraciones, es decir, representa la capacidad de la curva a adaptarse a los contornos a través de la imagen.

La fuerza de presión $F$, que regula el grado de dilatación o compresión de la curva, se expresa como:

$$
F=k \cdot \hat{a}(V)
$$

donde $\hat{a}(V)$ es el vector normal a la curva en cualquier punto $v_{i}$ y $k$ es un peso constante. El signo de $k$ determina si la curva tiende a dilatarse o a comprimirse.

La energía externa se determina a partir de las características de la imagen, de manera que toma los valores mínimos en los bordes de la imagen. En nuestro caso, se utilizó el GVF como energía externa. El modelo de GVF se basa en la creación de un campo de fuerzas a partir de la difusión del gradiente de un mapa de bordes sobre toda la imagen (Fig. 5c). La difusión se efectúa mediante una adaptación de la fuerza a los contornos de cada región, otorgando mayor peso a las zonas con más bordes y menor peso a aquellas que presenten menos bordes; es decir, cada punto del contorno deformable se verá empujado hacia el borde más fuerte. La imagen de entrada al algoritmo GVF fue la imagen de atlas binarizada e invertida (Fig. 5b).

Para minimizar la función de energía (7), la curva $V$ debe resolver la ecuación de Euler:

$$
\alpha V^{\prime \prime}-\beta V^{\prime \prime \prime}-\nabla E_{\text {ext }}=0
$$

donde $\nabla$ es el operador gradiente.

En el modelo de GVF, $-\nabla E_{e x t}$ se sustituye por $v\left(x_{i}, y_{i}\right)$, que es el campo de vectores que resuelve la ecuación de Euler:

$$
\mu \nabla^{2} v-(v-\nabla f)|\nabla f|^{2}=0
$$

donde $\nabla^{2}$ es el operador laplaciano, $\mu$ es un parámetro de regularización ajustado en función de la cantidad de ruido en la imagen (mayor ruido, mayor $\mu$ ) y $f$ es el mapa de bordes de la imagen, que en nuestro caso, al ser una imagen binaria, es:

$$
f(x, y)=-I(x, y)
$$

donde $I(x, y)$ es la imagen de atlas binarizada.

Otro de los parámetros (kappa, $\kappa$ en (7)) determina el peso de la fuerza externa, es decir, la importancia de la información de la imagen en el comportamiento de la curva.
Por último, el número de iteraciones determina la velocidad a la que el contorno inicial se adapta a las regiones definidas por el GVF: a mayor número de iteraciones, el proceso será más lento pero el contorno se ajustará de forma más precisa.

Resumiendo, los parámetros del modelo de contornos activos se presentan en la TABLA I. En la Fig. 5b se puede observar un ejemplo de adaptación automática del contorno, mediante el algoritmo GVF aplicado con los parámetros especificados.

TABLA I. PARÁMETROS DEL SNAKE.

\begin{tabular}{|l|c|l|c|}
\hline Alpha (elasticidad) & 1 & Presión & 0,01 \\
\hline Beta (rigidez) & 0,3 & Kappa (peso fuerzas externas) & 3,2 \\
\hline Gamma (viscosidad) & 2 & Iteraciones & 50 \\
\hline
\end{tabular}

\section{G. Reconstrucción tridimensional}

Tras obtener todos los contornos de la región que se iba a reconstruir, se procedió a la reconstrucción tridimensional. Se obtuvieron las máscaras correspondientes a dichos contornos y con ellas se extrajo la región en cada corte de imagen PET. Se utilizó la técnica de superposición de contornos, otorgando a cada imagen, es decir, a cada región, un espesor obtenido de las características del tomógrafo $(0,5 \mathrm{~mm}$ en este caso). Finalmente, se aplicaron las opciones gráficas para obtener un modelado tridimensional adecuado, con la posibilidad de aproximar o alejar el objeto de la reconstrucción así como de girar el mismo para cambiar el ángulo de visión y obtener distintas vistas de la representación 3D. En la Fig. 5e se muestra un ejemplo de reconstrucción tridimensional de la región correspondiente al cerebro. A partir de ésta se pueden ya realizar medidas cualitativas de la captación cerebral de ${ }^{18} \mathrm{~F}-\mathrm{FDG}$, que ayudan a la interpretación de los valores numéricos de cuantificación. La reconstrucción puede ser almacenada para análisis posteriores.

\section{H. Cuantificación de la captación cerebral de ${ }^{18} F-F D G$}

En nuestro estudio, se analizó la captación cerebral de ${ }^{18} \mathrm{~F}-$ FDG en el cerebro completo, centrando la ROI en el área central del cerebro expresado en centímetros cúbicos $\left(\mathrm{cm}^{3}\right)$, y excluyendo la parte correspondiente al cerebelo y el resto de las regiones con hipercaptación de la cabeza, como las glándulas de Harder.

Para obtener la actividad de ${ }^{18} \mathrm{~F}-\mathrm{FDG}$ en el volumen de interés, se extrajeron en primer lugar los valores de intensidad de píxel de la ROI definida en cada corte, obteniendo así los valores de intensidad de los píxeles del volumen de interés. A continuación se convirtieron estos valores en actividad de ${ }^{18} \mathrm{~F}$ FDG (o concentración de ${ }^{18} \mathrm{~F}-\mathrm{FDG}$ ) expresada en milicurios por unidad de volumen $\left(\mathrm{mCi} / \mathrm{cm}^{3}\right)$, como se describe en [22].

Esta actividad se corrigió por la dosis inyectada (mCi) y el peso del animal (g), obteniendo el SUV cerebral según (13): 


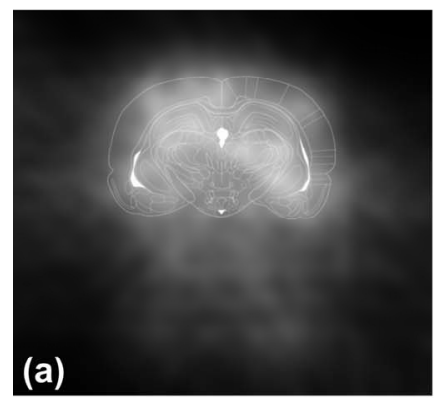

(b)

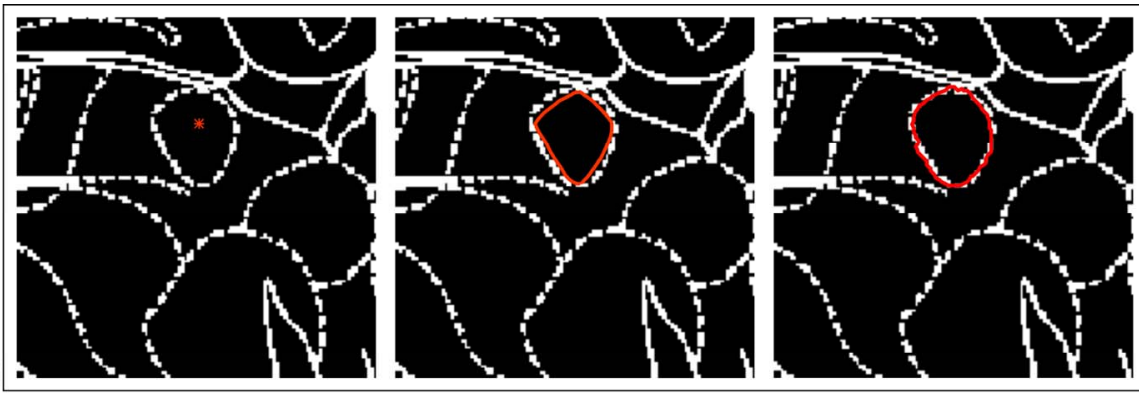

(c)
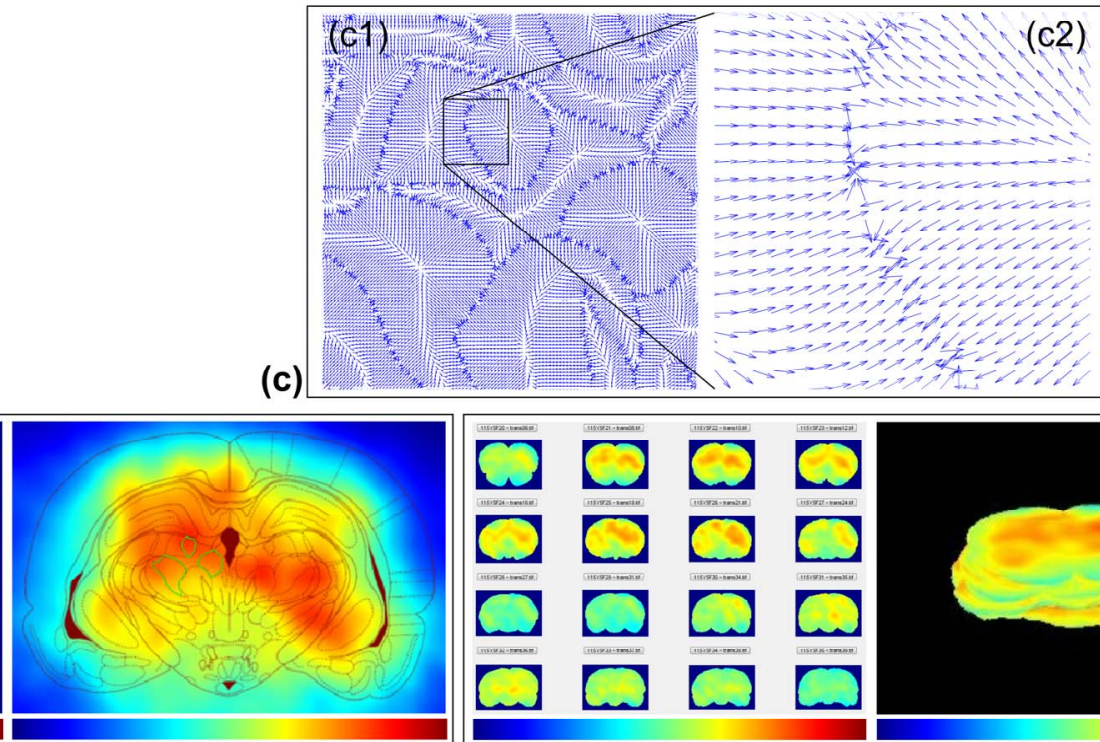

(e)

(d)

Figura 5. Descripción del proceso de análisis llevado a cabo por la herramienta. (a) Corregistro de las imágenes PET (Fig. 2a) y de atlas (Fig. 2b). (b,c) Segmentación de una ROI del atlas: (b) semilla inicial y adaptación automática del contorno a la ROI del atlas; (c) información de bordes de una imagen de atlas. Campo de fuerzas para toda la imagen (c1) y zoom del mismo (c2), donde se puede apreciar la convergencia hacia el contorno. (d) Resultados 2D de las distintas regiones seleccionadas. (e) Resultados 2D de la región del cerebro completo y reconstrucción 3D del mismo.

$$
S U V=\frac{\text { Actividad de }{ }^{18} F-F D G \text { en } R O I}{\frac{\text { Dosisinyectada }}{\text { Peso corporal }}}
$$

donde $R O I$ es el área de interés cerebral.

Se obtuvo el SUV cerebral en todos los animales estudiados, y se expresó el valor medio de SUV de acuerdo al sexo.

\section{Análisis estadístico}

Las variables dosis inyectada, peso corporal del animal, área de la ROI y SUV cerebral se expresaron como media \pm desviación típica. Para el análisis estadístico, se utilizó el test Least Significant Difference (LSD) que consiste en dos etapas: primero se realizó un análisis de varianza. La hipótesis nula fue aceptada para todos los valores de aquellos grupos en los cuales $F$ no fue significativa para un valor $p<0,05$. A continuación el grupo de datos para los que $\mathrm{F}$ fue significativa fueron analizados por el test $t$ de Student. Se consideraron significativos los valores de $\mathrm{p}<0,05$. Para el análisis estadístico, se utilizó el paquete estadístico SPSS 19.0 (IBM, Somers, NY, Estados Unidos de América).

\section{Resultados}

La aplicación de la herramienta a la población de ratas descrita ha permitido cuantificar el área cerebral de interés (ROI en $\mathrm{cm}^{3}$ ) y la correspondiente actividad de ${ }^{18} \mathrm{~F}-\mathrm{FDG}$ $\left(\mathrm{mCi} / \mathrm{cm}^{3}\right)$ en cada una de las ratas, obteniendo así los valores de SUV cerebral.

En primer lugar, se ha cuantificado el área de la ROI cerebral correspondiente a cada una de las ratas. En segundo lugar, se ha obtenido la actividad de ${ }^{18}$ F-FDG. Los valores de dosis inyectada, peso corporal, área de la ROI y la actividad de ${ }^{18}$ F-FDG figuran en la TABLA II.

En la TABLA III se muestra el área de la ROI en los grupos de ratas jóvenes y viejas y, como se puede comprobar, ésta disminuyó con la edad, tanto en machos como en hembras (macho: 2,52 $\pm 0,19$ vs. $1,75 \pm 0,14 \mathrm{~cm}^{3}, \mathrm{p}<0,05$; hembra: $2,62 \pm 0,04$ vs. $\left.1,73 \pm 0,06 \mathrm{~cm}^{3}, \mathrm{p}<0,05\right)$. 
TABLA II. DESCRIPCIÓN DE LAS VARIABLES DEL ESTUDIO.

\begin{tabular}{|l|c|c|c|c|}
\hline Variables & Media & $\begin{array}{c}\text { Desviación } \\
\text { típica }\end{array}$ & Máximo & Mínimo \\
\hline Dosis inyectada $(\mathrm{mCi})$ & 0,951 & 0,071 & 1,040 & 0,834 \\
\hline Peso $(\mathrm{g})$ & 273,4 & 90,3 & 400,0 & 157,0 \\
\hline ROI $\left(\mathrm{cm}^{3}\right)$ & 2,11 & 0,46 & 2,7 & 1,6 \\
\hline Actividad ${ }^{18} \mathrm{~F}-\mathrm{FDG}\left(\mathrm{mCi} / \mathrm{cm}^{3}\right)$ & 0,015 & 0,009 & 0,038 & 0,005 \\
\hline
\end{tabular}

mCi: milicurios; ROI $\left(\mathrm{cm}^{3}\right)$ : volumen de la ROI (en centímetros cúbicos).

TABLA III. ÁREA CEREBRAL DE INTERÉS (ROI) EN RATAS JÓVENES Y VIEJAS.

\begin{tabular}{|l|l|c|}
\hline \multicolumn{2}{|l|}{ ROI $\left(\mathbf{c m}^{\mathbf{3}}\right)$} & Media \pm Desviación Estándar \\
\hline \multirow{2}{*}{ Joven } & Macho & $2,52 \pm 0,19$ \\
\cline { 2 - 3 } & Hembra & $2,62 \pm 0,04$ \\
\hline \multirow{2}{*}{ Vieja } & Macho & $1,75 \pm 0,14^{*}$ \\
\cline { 2 - 3 } & Hembra & $1,73 \pm 0,06^{*}$ \\
\hline
\end{tabular}

ROI $\left(\mathrm{cm}^{3}\right)$ : volumen de la ROI (en centímetros cúbicos); * $\mathrm{p}<0,05$ vs. joven.

La Fig. 6 muestra el efecto del envejecimiento en el consumo cerebral de glucosa, así como las diferencias entre sexos. Al analizar los valores de SUV cerebral según género se observó que el SUV disminuyó de manera significativa $(\mathrm{p}<$ 0,05 ) con la edad, tanto en machos como en hembras (macho: $3,2 \pm 0,3$ vs. $2,4 \pm 0,0 \mathrm{SUV}, \mathrm{p}<0,05$; hembra: $5,1 \pm 1,0$ vs. $2,7 \pm 1,0 \mathrm{SUV}, \mathrm{p}<0,05)$. Por tanto, el envejecimiento interfiere con el consumo cerebral de glucosa en las ratas. Estos resultados corroboran estudios previos [9], [10], lo que muestra la precisión de la herramienta desarrollada en el cálculo de la captación cerebral de glucosa.

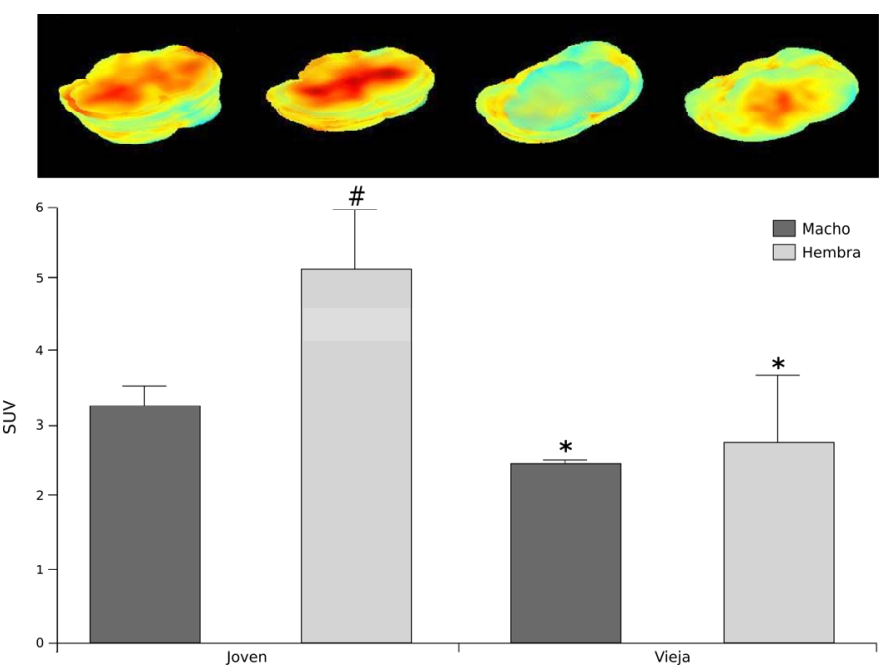

Figura 6. Consumo cerebral de glucosa in vivo en ratas jóvenes (4-7 meses) y viejas (22-24 meses). Las imágenes son el resultado de la reconstrucción 3D de 4 experimentos representativos (macho joven, hembra joven, macho viejo, hembra vieja). El histograma representa el metabolismo cerebral de glucosa ( ${ }^{18}$ F-FDG) expresado como Standardized Uptake Value (SUV). Los valores están expresados como media \pm desviación estándar. La diferencia estadística se expresa como $* \mathrm{p}<0,05$ vs. joven; $\# \mathrm{p}<0,05$ vs. macho.

Además, en el grupo de ratas jóvenes, el SUV cerebral fue mayor en las ratas hembra $(3,2 \pm 0,3$ vs. $5,1 \pm 1,0 \mathrm{SUV}, \mathrm{p}<$ $0,05)$, mientras que con el envejecimiento dichas diferencias desaparecieron ( $2,4 \pm 0,0$ vs. $2,7 \pm 1,0 \mathrm{SUV}$, ns).

Los resultados obtenidos demuestran por tanto la capacidad de la herramienta desarrollada para poder definir rangos de variación de la captación de glucosa en una determinada población de roedores de forma automática, mostrando además la sensibilidad al sexo del roedor, cumpliendo con los criterios de significación estadísticos y con una buena reproducibilidad de la metodología.

\section{CONCLUSIONES}

Se ha presentado una herramienta-software que permite la cuantificación semi-automática de glucosa en el cerebro de rata mediante el corregistro de las imágenes PET con un atlas anatómico estandarizado, del que se obtiene la información anatómica necesaria para localizar las regiones de interés e identificarlas. La aplicación permite asimismo la generación automática de informes a partir de las medidas realizadas, el almacenamiento de los resultados de los estudios realizados para su posterior consulta y la exportación de dichos resultados para análisis posteriores.

La herramienta permite obtener resultados precisos y objetivos, logrando definir rangos de variación del consumo cerebral de glucosa en una población de roedores jóvenes y viejos (ratas), cumpliendo los criterios de significación estadística. Estos resultados muestran un descenso con el envejecimiento del consumo cerebral de glucosa en ratas macho y hembra, y corroboran por tanto datos publicados previamente [9], [10] de estudios realizados en ratones de un modelo de envejecimiento acelerado y en ratas Wistar hembra.

La accesibilidad que ofrece la herramienta y la calidad en la presentación de los resultados hacen de esta aplicación una herramienta útil y con un potencial muy interesante para asistir al investigador biomédico en la realización de análisis más objetivos y precisos, reduciendo el error de variabilidad del usuario asociado a la selección manual de las ROIs, así como en la interpretación de los resultados obtenidos.

La herramienta desarrollada posee posibles aplicaciones en el estudio de enfermedades neurodegenerativas como la demencia tipo Alzheimer o en el ámbito de la neurooncología. En el primer caso, permitiría obtener el metabolismo glucídico del cerebro completo o de distintas regiones cerebrales, en las que se conoce o se supone un deterioro causado por la enfermedad. En el segundo caso, permitiría el diagnóstico diferencial de masas intracraneales, donde una gran variedad de lesiones comparten la misma apariencia morfológica y la diferenciación entre proceso tumoral, infeccioso, absceso o necrosis puede resultar complejo mediante la TC (Tomografía Computarizada) o la RMN (Resonancia Magnética Nuclear) [5]. Por ello, se plantea la utilización de esta herramienta para diferenciar las regiones en las que hay células tumorales viables (hipermetabolismo) de las regiones de necrosis postradiación (generalmente hipometabólicas).

Las principales limitaciones son la velocidad y la precisión del corregistro entre las imágenes PET y el atlas. Con el fin de 
reducir dicha deficiencia, se han propuesto futuras ampliaciones de la herramienta. Éstas se basarán en la fusión de las técnicas PET/TC [23] o PET/RMN [24], lo que permitirá un corregistro automático del atlas con la imagen de TC o de RMN, y por tanto el corregistro automático del atlas con la imagen de PET.

\section{REFERENCIAS}

[1]A. Loessner, A. Alavi, K. U. Lewandrowski, D. Mozley, E. Souder and R. E. Gur, "Regional cerebral function determined by FDG-PET in healthy volunteers: Normal patterns and changes with age", J Nucl Med, 36, pp. 1141-1149, 1995.

[2] S. Minoshima, B. Giordani, S. Berent, K. A. Frey, N. L. Foster and D. E. Kuhl, "Metabolic reduction in the posterior cingulate cortex in very early Alzheimer's disease". Ann Neurol, 42, pp. 85-94, 1997.

[3]L. Mosconi, R. Mistur, R. Switalski, M. Brys, L. Glodzik, K. Rich, E. Pirraglia, W. Tsui, S. De Santi and M. J. de Leon, "Declining brain glucose metabolism in normal individuals with a maternal history of Alzheimer disease", Neurology, 72, pp. 513-520, 2009.

[4]C. Huang, L. D. Ravdin, M. J. Nirenberg, P. Piboolnurak, L. Severt, J. S. Maniscalco, L. Solnes, B. J. Dorfman and C. Henchcliffe, "Neuroimaging markers of motor and nonmotor features of Parkinson's disease: an $18 \mathrm{f}$ fluorodeoxyglucose positron emission computed tomography study", Dement Geriatr Cogn Disord, 35, pp. 183-196, 2013.

[5]C. Colavolpe, O. Chinot, P. Metellus, J. Mancini, M. Barrie, C. BequetBoucard, E. Tabouret, O. Mundler, D. Figarella, D. Branger and E. Guedj, "FDG-PET predicts survival in recurrent high-grade gliomas treated with bevacizumab and irinotecan", Neuro Oncol, 14, pp. 649-657, 2012.

[6]M. J. De León, A. Convit, O. T. Wolf, C. Y. Tarshish, S. De Santi, H. Rusinek, W. Tsui, E. Kandil, A. J. Scherer, A. Roche, A. Imossi, E. Thorn, M. Bobinski and C. Caraos, "Prediction of cognitive decline in normal elderly subjects with 2-[18F]fluoro- 2-deoxy-D-glucose/positron-emission tomography (FDG/PET)", Proc Natl AcadSci USA, 98, pp. 10966-10971, 2001.

[7] J. V. Pardo, J. T. Lee, S. A. Sheikh, C. Surerus-Johnson, H. Shah, K. R. Munch, J. V. Carlis,S. M. Lewis, M. A. Kuskowski and M. W. Dysken, "Where the brain grows old: Decline in anterior cingulate and medial prefrontal function with normal aging", Neuroimage, 35, pp. 1231-1237, 2007.

[8]L. Mosconi, S. De Santi, J. Li, W. H. Tsui, Y. Li, M. Boppana, E. Laska, H. Rusinek and M. J. de Leon, "Hippocampal hypometabolism predicts cognitive decline from normal aging", Neurobiol Aging, 29, pp. 676-692, 2008.

[9]C. Borrás, S. Stvolinsky, R. López-Grueso, T. Fedorova, J. Gambini, A. Boldyrev, J. Viña. "Low in vivo brain glucose consumption and high oxidative stress in accelerated aging", FEBS Lett, 583, pp. 2287-2293, 2009.

[10] R. López-Grueso, C. Borrás, J. Gambini and J. Viña, "Aging and ovariectomy cause a decrease in brain glucose consumption in vivo Wistar rats", Rev Esp Geriatr Gerontol, 45, pp. 136-140, 2010.

[11] Grupo PET de la SEMM, "Tomografía de positrones (PET) de cuerpo entero con fluordesoxiglucosa-18F - Whole body positron emission tomography (PET) with 18F-fluordesoxyglucose", Rev Esp Med Nucl, 21, pp. 128-130, 2002.

[12] M. D. Silva, C. Glaus, J. Y. Hesterman, J. Hoppin, G. H. Puppa, T. Kazules, K. M. Orcutt, M. Germino, D. Immke and S. Miller, "Regional, kinetic [(18)F]FDG PET imaging of a unilateral Parkinsonian animal model", Am J Nucl Med Mol Imaging, 3, pp. 129-141, 2013.

[13] A.J. Reader and H. Zaidi, "Advances in PET Image Reconstruction", PET Clin, 2, pp. 173-190, 2007.

[14] S. Yamaji, K. Ishii, M. Sasaki, T. Mori, H. Kitagaki, S. Sakamoto and E. Mori, "Evaluation of standardized uptake value to assess cerebral glucose metabolism", Clin Nucl Med, 25, pp. 11-16, 2000.

[15] BOE, "Real Decreto 1201/2005, de 10 de octubre, sobre protección de los animales utilizados para la experimentación y otros fines científicos", BOE, 252, pp. 34367-34391, 2006.

[16] B. J. Fueger, J. Czernin, I. Hildebrandt, C. Tran, B. S. Halpern, D. Stout, M. E. Phelps and W. A. Weber, "Impact of animal handling on the results of 18F-FDG PET studies in mice", J Nucl Med, 47, pp. 999-1006, 2006.

[17] K. Hamacher, H. H. Coenen and G. Stocklin, "Efficient stereospecific synthesis of no-carrier-added 2-[18F]-fluoro-2-deoxy-D-glucose using aminopolyether supported nucleophilic substitution”, J Nucl Med, 27, pp. 235$238,1986$.

[18] C. W. Lerche, J. M. Benlloch, F. Sanchez, N. Pavon, B. Escat, E. N. Giménez, A. Sebastia and J. Martinez, "Depth of gamma-ray interaction within continuous crystals from the width of its scintillation lightdistribution", IEEE T Nucl Sci, 52, pp. 560-72, 2005.

[19] Paxinos G and Watson C, The rat brain in stereotaxic coordinates, 4th edition. San Diego: Academic Press, 1998.

[20] M. Kass, A. Witkin and D. Terzopoulos, "Snakes: active contour models", Int J Comput Vis, 1, pp. 321-331, 1988.

[21] C. Xu and J. L. Prince, "Snakes, shapes and gradient vector flow", IEEE Trans Image Process, 7, pp. 359-369, 1998.

[22] S. Soongsathitanon, M. Tuntawiroon, T. Thongpraparn and P. Masa-Ah, "Performance of a novel SUV calculation scheme for PET study", Recent advances in applied \& biomedical informatics and computational engineering in systems applications, WSEAS, pp. 83-87, 2011.

[23] F. P. DiFilippo, S. Patel, K. Asosingh and S. C. Erzurum, "Small-animal imaging using clinical positron emission tomography/computed tomography and super-resolution", Mol Imaging, 11, pp. 210-219, 2012.

[24] H. Herzog, "PET/MRI: challenges, solutions and perspectives", Z Med Phys, 22, pp. 281-298, 2012.

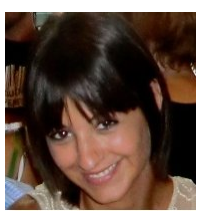

Irene Del-Canto nació en Valencia, España, en 1984. Obtuvo el título de Ingeniería de Telecomunicación en la Universitat Politécnica de València (UPV) en 2010. Cursó también el Máster en Ingeniería Biomédica (Universitat Politècnica de València y Universitat de València), realizando su Trabajo de Fin de Máster sobre la cuantificación de imágenes obtenidas mediante la técnica PET (Positron Emission Tomography). Actualmente es Investigadora en la Fundación de Investigación del Hospital Clínico Universitario de Valencia, INCLIVA, en el Grupo de Electrofisiología Cardiaca Experimental, y realiza su doctorado en Tecnologías para la Salud y el Bienestar en la UPV. Su investigación se centra en el tratamiento de señales de origen cardiaco multicanal y en el estudio y análisis de los mecanismos intramiocárdicos a partir de imágenes de Resonancia Magnética Cardiaca con tagging.

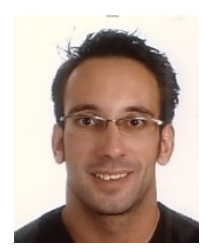

Raúl López-Grueso nació en Las Navas de la Concepción, Sevilla, España, en 1980. Se licenció en Ciencias del Deporte en el año 2002 en Granada. También se diplomó en Nutrición humana y dietética (2005) y comenzó su doctorado en nutrición humana (2002) con el Dr. Guerra en Universidad de Granada (Departamento de nutrición y Bromatología) investigando sobre la obesidad y sus factores de riesgo. Obtuvo una beca de investigación del Ministerio de Educación y Ciencia para realizar la tesis con el Dr. Viña en el Departamento de Fisiología de la Universidad de Valencia en 2006, centrando su investigación en la técnica PET (Positron Emission Tomography) y su interés para estudiar el metabolismo de la glucosa (FDG) en situaciones fisiológicas (i.e: envejecimiento, género, ejercicio, etc.) y patológicas (i.e: cáncer, enfermedades Alzheimer's, etc..). Actualmente, es profesor ayudante doctor en la Universidad Miguel Hernández (Elche, Alicante) impartiendo asignaturas en el Grado de Ciencias de la Actividad Física y del Deporte y en el Máster en Rendimiento Deportivo y Salud; y realiza sus trabajos de investigación en el Centro de Investigación del Deporte, concretamente en el Grupo de Investigación en Acondicionamiento Físico Saludable.

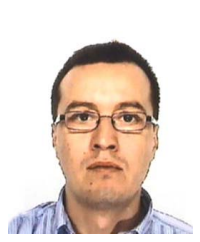

Juan Gambini se licenció en Farmacia en 2001 en la Universidad de Valencia. Finalizó su tesis doctoral en el laboratorio de José Viña, del Departamento de Fisiología de la Universidad de Valencia en 2007, y dos años más tarde le otorgaron mención especial. Durante su periodo de estudiante de doctorado, realizó una estancia pre-doctora en Dallas (EEUU) en la SMU University con el Dr. Bill Orr y en Düsseldorf (Alemania) en la Universidad de Düsseldorf con el Dr. Klotz y Dr. Sies. Actualmente, es profesor ayudante doctor de Fisiología en la Universidad de Valencia. Las líneas de investigación en las que trabaja se centran en la longevidad, concretamente en las modificaciones que ejerce la nutrición en los genes de la longevidad y en el estrés oxidativo. 


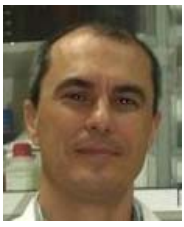

Daniel Monleón obtuvo la licenciatura (1993) y el doctorado (1998) en Química en la Universidad de Valencia. Desde 2004, su investigación se centra en los perfiles moleculares (metabolómica, imagen molecular, transcriptómica, etc.) y el análisis multivariable para aplicaciones biomédicas y clínicas (cáncer, enfermedad cardiovascular). Actualmente trabaja en la Universitat de València como profesor asociado del Departamento de Patología, impartiendo las asignaturas de Embriología, Biología, Oncología y Morfología a nivel celular y tisular. Desde 2007, dirige la Unidad de Imagen Molecular y Metabolómica de la Fundación de Investigación del Hospital Clínico Universitario de Valencia (INCLIVA), que centra su investigación en la búsqueda de biomarcadores y factores de riesgo de distintas patologías, así como en el desarrollo de herramientas y metodologías para su aplicación en la rutina clínica.

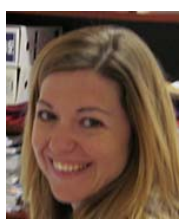

Consuelo Borrás nació en Valencia, España, en 1976. Se licenció en Farmacia en 1999 en la Universidad de Valencia. Posteriormente obtuvo un doctorado europeo en fisiología (2003) en el Departamento de Fisiología en la Facultad de medicina de la Universidad de Valencia con el título "La importancia del estrés oxidativo en la diferencia de longevidad entre machos y hembras" supervisada por el Prof. José Viña. A raíz de una posición como técnico superior de investigación en la Universidad de Valencia y una plaza de profesora de Fisiología en la Universidad Católica de Valencia, fue nombrada en 2008 profesor contratado doctor de Fisiología en la Facultad de Medicina de la Universidad de Valencia. Desde 2011, es profesora titular de Fisiología de la Universidad de Valencia, y sus líneas de investigación se centran en el estudio de los factores que confieren longevidad extrema a los centenarios, así como el estudio de las células madres como herramienta para regenerar tejidos envejecidos.

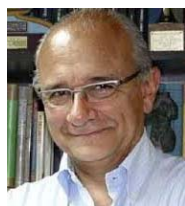

José Viña nació en Valencia, España, en 1953. Después de cursar sus estudios en Medicina en la Universidad de Valencia, y realizar su trabajo de investigación bajo los auspicios del profesor Hans Krebs (ciclo de Krebs) en Oxford, obtuvo su doctorado en 1976. Tras enseñar Fisiología en la Universidad de Extremadura (España) volvió a Valencia y tomó su puesto como profesor de Fisiología en la Universidad de Valencia, donde aún trabaja. En dicha universidad, el Prof. Viña combina sus tareas de enseñanza con el trabajo de investigación, este último centrado en dos líneas principales: envejecimiento y ejercicio. José Viña dirige un grupo de investigación exitoso sobre diferentes aspectos del estrés oxidativo con quien ha ganado numerosos premios por trabajos de investigación; ha publicado de forma extensa sobre glutatión, mitocondrias, estrés oxidativo, radicales libres y nutrición.

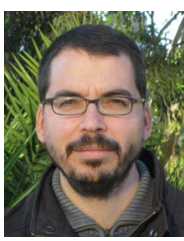

David Moratal (SM'13) nació en Gandía (Valencia), España, en 1976. En el año 2001 recibió el título de Ingeniero de Telecomunicación por la Universitat Politècnica de València (España) y el de Ingénieur Supélec por la École Supérieure d'Électricité (Francia). En 2006 obtuvo el grado de Doctor (Doctorado Europeo) por la Universitat Politècnica de València. Actualmente es profesor Titular de Universidad en el Departamento de Ingeniería Electrónica de la Universitat Politècnica de València (España) y desde el año 2008 es miembro del Centro de Biomateriales e Ingeniería Tisular de esta misma Universidad. Su campo de interés es el procesado y análisis de imágenes biomédicas así como el estudio de las distintas técnicas de adquisición y reconstrucción de imágenes médicas. El Dr. Moratal es miembro del IEEE, la ESMRMB y la ISMRM, y es autor o coautor de más de 70 artículos científicos y de varios libros y capítulos de libro. 